\title{
A comparison of spinal anesthesia characteristics following intrathecal bupivacaine or levobupivacaine in lumbar disc surgery
}

\author{
Ayça Sultan Şahin • Gürkan Türker • \\ Ahmet Bekar · Hülya Bilgin · Gülsen Korfalı
}

Received: 5 November 2012/Revised: 24 October 2013/ Accepted: 26 October 2013/Published online: 9 November 2013

(C) Springer-Verlag Berlin Heidelberg 2013

\begin{abstract}
Purpose While bupivacaine is the most frequently used local anesthetic for spinal anesthesia, use of levobupivacaine in clinical practice has advanced recently. The aim of our study was to compare the clinical and anesthetic effects of isobaric bupivacaine and isobaric levobupivacaine when administered intrathecally in patients undergoing lumbar disc surgery.

Methods ASA I-III, 60 patients were enrolled in this study. Only patients with unilateral single-level (L4-5) lumbar disc hernia were selected and operated in each group and all were operated by the same surgeon. Patients were randomized into two groups, as group B $(n=30)$ : $15 \mathrm{mg} 0.5 \%$ isobaric bupivacaine, or group $\mathrm{L}(n=30)$ : $15 \mathrm{mg} 0.5 \%$ isobaric levobupivacaine received intrathecally. The level of sensory block dermatome, degree of motor block, intraoperative sensory and motor block characteristics, and postoperative recovery times of spinal anesthesia were evaluated. The satisfaction scores of the surgeon and patients, intraoperative hemodynamic
\end{abstract}

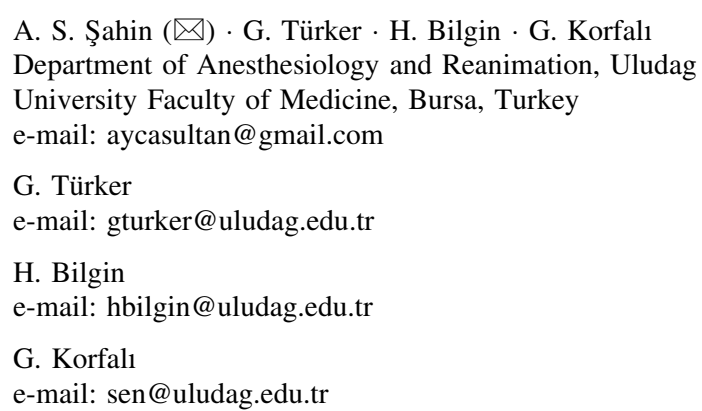

A. Bekar

Department of Neurosurgery, Uludag University Faculty of Medicine, Bursa, Turkey

e-mail: abekar@uludag.edu.tr changes, intraoperative and postoperative complications were recorded.

Results The maximum level of sensory blockade was significantly higher in the levobupivacaine group (group L $7 \pm 1.63$, group B $8.6 \pm 1.76$ thoracic dermatome, $p<0.05)$. There was no significant difference in the onset time of sensory (group L $6 \pm 3 \mathrm{~min}$, group B $9 \pm 4 \mathrm{~min}$ ) and motor (in group L $7 \pm 3 \mathrm{~min}$, in group B $10 \pm 4 \mathrm{~min}$ ) blockade $(p>0.05)$. There was no significant difference between the groups regarding duration of operation (group L $49 \pm 7.3 \mathrm{~min}$, group B $52 \pm 8.1, p>0.05$ ). Recovery times of sensory $(175 \pm 57 \mathrm{~min})$ and motor $(216 \pm 59 \mathrm{~min})$ blockade were significantly shorter in the levobupivacaine group $(p<0.05)$. Mobilization was also earlier in the levobupivacaine group $(339 \pm 90 \mathrm{~min}, p<0.05)$. Patients' satisfaction and intraoperative, postoperative complications were similar between the two groups.

Conclusions Our results showed that block recovery time was shorter in the levobupivacaine group, this may be a disadvantage for longer operative procedures. But with proper patient selection this can be eliminated. Recovery time was shorter in levobupivacaine group. Therefore, postoperative neurological examination can be done earlier. In addition, early mobilization can be an advantage for postoperative recovery.

Keywords Spinal anesthesia - Bupivacaine . Levobupivacaine

\section{Introduction}

Procedures on the lumbar spine vary in complexity from simple discectomy to complex procedures. Many anesthesiologists prefer general anesthesia in the prone position 
because spinal anesthesia has a limited duration and variation in operative times which may result the anesthesiologist having to start a general anesthesia in the prone position with manipulation of the airway. Spinal anesthesia has been reported with good success for elective lumbar spine procedures [1]. Studies suggest that spinal anesthesia reduces blood loss, decreased early postoperative pain, lower incidence nausea and vomiting [2]. The patients can change their positions while under spinal anesthesia; and can move their extremities and chest to avoid nerve injury, brachial plexus palsy, or pressure necrosis to either the face or the chest wall $[3,4]$. Spinal techniques may also reduce the incidence of pulmonary complications compared with general anesthesia [5]. Some surgeons and/or patients prefer general anesthesia for their comfort, but recently, both anesthesiologists and surgeons have preferred spinal anesthesia in compatible and selected patients who have no need for general anesthesia (e.g. in unilateral/single-level discectomy with a short surgical time).

Hyperbaric local anesthetic solutions are generally used in spinal anesthesia, but in the prone position, it may cause the spread of the local anesthetic solution in a cephalic direction and produce motor blockade in the anterior roots of the thoracic region, leading to ventilatory depression [6]. In the prone position, local anesthetic concentration in the anterior spinal segments increases over time and motor blockade also increases. Conversely, local anesthetic concentration in posterior segments decreases and sensory blockade may become insufficient over time. Patient may feel pain despite being unable to move their limbs. Thus, hyperbaric local anesthetic is usually not used for spinal anesthesia in the prone position [7]. For these reasons, we used isobaric bupivacaine and levobupivacaine in our study.

Levobupivacaine is a long-acting amide local anesthetic that is effective when administered as an epidural, spinal, peripheral nerve, or ocular block, or by topical application or local infiltration. In comparative trials, its clinical effects were not generally significantly different from those of bupivacaine, although there was some variability in efficacy findings in different clinical populations. Levobupivacaine provides effective anesthesia and analgesia for a wide range of clinical populations and is a useful alternative to bupivacaine [8].

The aim of our study was to compare the clinical and anesthetic effects of isobaric bupivacaine and isobaric levobupivacaine when intrathecally administered to patients undergoing lumbar disc surgery.

\section{Methods}

Following approval of the local Ethics Committee, 60 patients aged 20-80 years, with ASA I-III physical status, who were scheduled for elective lumbar disc surgery with spinal anesthesia, were enrolled into the study. In our study, only patients with unilateral single-level (L4-5) lumbar disc hernia were selected and operated in each group, and the same surgeon performed all the operations. The patients with medical contraindication to spinal anesthesia (allergy, bleeding disorders, localized infection or neurological disease) were excluded from the study.

Patients were randomly allocated into one of the two groups for spinal anesthesia according to numbers inserted into sealed envelopes. Following the application of routine monitoring and the infusion of sodium chloride $0.9 \%$ solution, baseline hemodynamic values were recorded and then spinal anesthesia was performed with the patient in the left lateral decubitus position, with a 25 gauge Quincke needle at the L3-4 interspace using a midline approach. The anesthesiologist, surgeon and recovery room nurse were unaware of which drug had been used because solutions were prepared by another anesthesiologist. Same anesthesiologist and same surgeon participated in this study. In group $\mathrm{L}(n=30), 3 \mathrm{ml}$ of $0.5 \%$ levobupivacaine, and in group B $(n=30) 3 \mathrm{ml}$ of $0.5 \%$ bupivacaine were administered intrathecally. After injection, patients turned to the supine position immediately. Sensory and motor blockade were assessed at 1, 3, 6, 9, 12 and 15 min (min) after time 0 , and then sensory blockade was assessed every 5 min using a pin-prick test (0: no sensory block, 1 : analgesia, 2: anesthesia) and motor blockade was assessed using the Bromage scale (0: no motor block, 1: ability to move knees only, inability to raise extended legs, 2: ability to move feet only, inability to flex knees, 3: full motor block) [9]. With respect to the surgical position, the patients were placed into the prone (knee-elbow) position, when pin-prick test was 2 .

The onset time of sensory blockade, the highest level of sensory block (dermatomes), and the time to two-segment regression of sensory blockade were recorded. The incidence of motor blockade at the beginning and at the end of surgery was recorded. Preoperative $0.03 \mathrm{mg} / \mathrm{kg}$ intravenous (iv) midazolam was given all patients for sedation. Heart rate (HR), non-invasive systolic, diastolic and mean arterial blood pressures (SAP, DAP, MAP) and oxygen saturation $\left(\mathrm{SpO}_{2}\right)$ were recorded at $1,3,6,9,12$ and 15 min after time 0 and then every $5 \mathrm{~min}$ thereafter. A $20 \%$ decrease from baseline SAP or SAP $<90 \mathrm{mmHg}$ was treated using incremental boluses of iv ephedrine $10 \mathrm{mg}$. Bradycardia $(\mathrm{HR}<45)$ was treated using iv atropine $0.5 \mathrm{mg}$. A decrease in $\mathrm{SpO}_{2}$ to $<90 \%$ was defined as hypoxemia and treated with supplemental oxygen via face mask. Side effects such as hypotension, bradycardia, nausea, and vomiting were also recorded. Pain was assessed from the beginning of surgery using a $10-\mathrm{cm}$ visual analog scale (VAS). 
During the postoperative period, one of the anesthesiologist who was unaware of the prepared local anesthetic checked the patients regarding sensory and motor blockade recovery times, and patients were asked whether they were satisfied or not with the technic use (0-3: 3, perfect; 2, some feelings but no discomfort; 1 , some discomfort but analgesic unnecessary; 0, major discomfort). In addition, the surgeon was asked to comment on the operating conditions (0-3: 0: very bad, 1: bad, 2: good, 3: very good). The patients were checked at 10-min intervals in the recovery room until recovery of $\mathrm{S} 2$ sensation. The patients were asked to report backache, headache or any transient neurological symptoms following the surgery. Diclofenac sodium $75 \mathrm{mg}$ intramuscular was administered when the patients complained of pain in the postoperative period, and it was sufficient in all patients.

All of the data were analyzed using Statistic Package for Social Sciences ver. 14.0 program. Statistical analyses were performed using Kruskal-Wallis and Mann-Whitney $U$ test between groups and Wilcoxon Signed Ranks test for each group. Data are presented as mean (SD), median (25-75th percentile), and number (incidence) as appropriate. Statistical significance was set at the $p<0.05$ level.

\section{Results}

Demographic data and vital signs

Sixty patients were recruited (levobupivacaine group $n=30$, bupivacaine group $n=30$ ). There were no significant differences between the levobupivacaine and bupivacaine groups with respect to demographic data, ASA classification (Table 1), hemodynamic parameters (MAP, $\mathrm{SpO}_{2}, \mathrm{HR}$ ), or type of operation.

Side effects of anesthesia were few and minor. The incidence of hypotension was $3.3 \%$ (2/60), and was noted in one patient from each of the two groups. Anesthesia was adequate in all patients, and none of patient needs general anesthesia or airway management. Two patients in the bupivacaine group experienced pain during the

Table 1 Demographic characteristics of the two groups

\begin{tabular}{lll}
\hline & Bupivacaine $(n=30)$ & Levobupivacaine $(n=30)$ \\
\hline Age (year) & $47 \pm 12$ & $44 \pm 11$ \\
Sex (F/M) & $16 / 14$ & $19 / 11$ \\
Weight $(\mathrm{kg})$ & $74 \pm 12$ & $75 \pm 15$ \\
Height $(\mathrm{cm})$ & $168 \pm 8$ & $167 \pm 10$ \\
ASA I/II/III & $20 / 9 / 1$ & $22 / 7 / 1$
\end{tabular}

Data are mean (SD) or number of patients (incidence)

ASA American Society of anesthesiologists intraoperative period. A female patient had a positional complaint of breast pain. None of the patients in either group received any additional sedatives or analgesics other than the standard regimen noted in the methods. Two patients in the bupivacaine group had nausea, and 1 patient had vomiting in postoperative period. None of patients had post-spinal headache or lumbar pain (Table 2).

Spinal anesthesia characteristics

The maximum level of sensory blockade (dermatomes) was significantly higher in the levobupivacaine group than in the bupivacaine group (group $\mathrm{L} 7 \pm 1.63$, group B $8.6 \pm 1.76$ thoracic dermatome, $p<0.05$ ) (Fig. 1). There was no significant difference in the onset time of sensory (group L $6 \pm 3 \mathrm{~min}$, group B $9 \pm 4 \mathrm{~min}$ ) and motor (group L $7 \pm 3 \mathrm{~min}$, group B $10 \pm 4 \mathrm{~min}$ ) blockade $(p>0.05)$. There was no significant difference between operative durations (group L $49 \pm 7.3 \mathrm{~min}$, group B $52 \pm 8.1, \quad p>0.05)$. Recovery times of sensory $(175 \pm 57 \mathrm{~min})$ and motor $(216 \pm 59 \mathrm{~min})$ blockade were significantly shorter in levobupivacaine group, $(p<0.05)$. The time to two-segment regression of sensory blockade was earlier in levobupivacaine group than in the bupivacaine group $(p>0.05)$. Mobilization of patients in

Table 2 Intraoperative and postoperative complications related with anesthesia

\begin{tabular}{lll}
\hline & $\begin{array}{l}\text { Bupivacaine } \\
(n=30)\end{array}$ & $\begin{array}{l}\text { Levobupivacaine } \\
(n=30)\end{array}$ \\
\hline Hypotension & 1 & 1 \\
Bradycardia & 0 & 0 \\
Nausea or vomiting & 3 & 1 \\
Urinary retention & 3 & 2 \\
Neurological complications & 0 & 0 \\
Headache & 0 & 0 \\
Lumbar pain & 0 & 0 \\
\hline
\end{tabular}

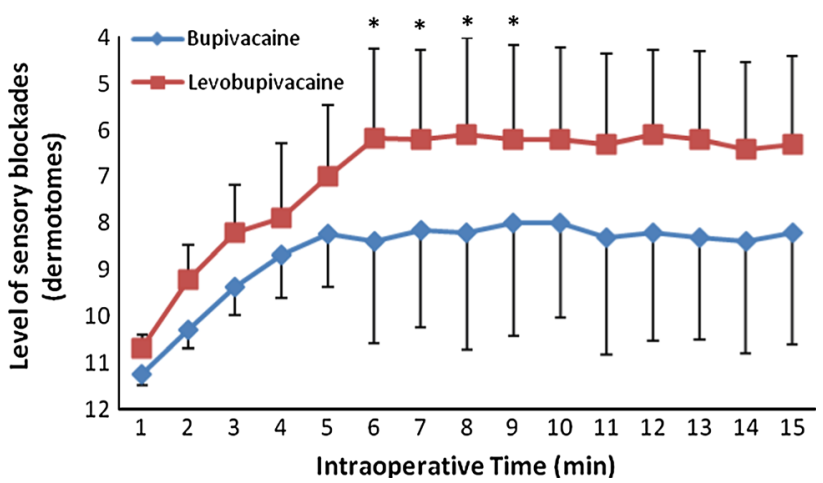

Fig. 1 The maximum level of sensory blockade (dermatomes). Data are mean $(\mathrm{SD})(* p<0.05)$ 
Table 3 Comparison of the spinal blockades

\begin{tabular}{lcc}
\hline & $\begin{array}{l}\text { Bupivacaine } \\
(n=30)\end{array}$ & $\begin{array}{l}\text { Levobupivacaine } \\
(n=30)\end{array}$ \\
\hline $\begin{array}{l}\text { Onset time of sensory } \\
\text { blockade (min) }\end{array}$ & $9 \pm 4$ & $6 \pm 3$ \\
$\begin{array}{l}\text { Onset time of motor blockade } \\
(\text { min) }\end{array}$ & $10 \pm 4$ & $7 \pm 3$ \\
$\begin{array}{l}\text { Time to two-segment } \\
\text { regression (min) }\end{array}$ & $103 \pm 35$ & $88 \pm 32^{*}$ \\
$\begin{array}{l}\text { Recovery time of sensory } \\
\text { blockade (min) }\end{array}$ & $266 \pm 112$ & $175 \pm 57^{*}$ \\
$\begin{array}{l}\text { Recovery time of motor } \\
\text { blockade (min) }\end{array}$ & $293 \pm 107$ & $216 \pm 59^{*}$ \\
\begin{tabular}{l} 
Time to mobilization (min) \\
\hline
\end{tabular} & $454 \pm 119$ & $339 \pm 90^{*}$ \\
\hline
\end{tabular}

Data are mean $(\mathrm{SD})$

* Statistical significance was set at the $p<0.05$ level

levobupivacaine group were earlier than in bupivacaine group (339 $\pm 90 \mathrm{~min}, p<0.05$ ) (Table 3 ). In addition, the surgeon felt more comfortable in levobupivacaine group. Surgical satisfaction was assessed as 3 in $90 \%$ of the cases in the levobupivacaine group, while in the bupivacaine group, this ratio was found to be $60 \%(p<0.05)$. The patients' satisfaction scores were similar in the two groups $(p>0.05)$. Intraoperative and postoperative complications were also similar between the two groups.

\section{Discussion}

In our study, we aimed to compare the intrathecal administration of $15 \mathrm{mg}$ isobaric levobupivacaine or $15 \mathrm{mg}$ isobaric bupivacaine in two groups regarding intraoperative sensory and motor blockade characteristics and postoperative recovery times from spinal anesthesia.

As all the patients in this study were placed in the prone position, we used isobaric local anesthetic solutions to minimize the degree of cephalic spread of the local anesthetic within the cerebrospinal fluid and none of our patients experienced ventilatory depression.

The majority of elective lumbar spine surgical procedures are performed with general anesthesia, a fact that is undoubtedly related to custom and a desire to secure the airway for procedures planned in the prone position [10]. During spinal anesthesia for longer operative procedures or highly variable operative times where institution of deep sedation and/or general anesthesia may be limited because of limited access to the airway. Longer blocks would provide less risk of conversion to a general anesthesia in the prone position if surgical times vary widely. But shorter blocks provide early recovery and nerologic examination. In our study, patients with L4-5 lumbar disc hernia were selected. Adequate levels of sensory analgesia were optioned in all patients before the surgery. The operation times were adequate (approximately $1 \mathrm{~h}$ ) for the surgical procedure. With proper patient selection, and careful surgeon requiring conversion to general anesthesia was none. We think that levobupivacaine may be preferred when the surgical times around $1 \mathrm{~h}$ but bupivacaine may be more appropriate if surgical times tend to be greater than $1 \mathrm{~h}$.

In this study, after injection of local anesthetics, hypotension was recorded in two patients, one in each group. There were no significant differences between the two groups in hypotension degree and frequency. Two patients in the bupivacaine group had nausea, and one patient had vomiting in the postoperative period. None of the patients had post-spinal headaches or lumbar pains.

In spinal anesthesia various needle types and local anesthetic have been used, differences in the outcome of the spinal anesthesia were determined more by the drugs than the equipment [10]. In our study, we used the same needles but two different drugs in all patients.

An advantage of spinal anesthesia is the patients' ability to reposition their extremities and chest to avoid nerve injury, brachial plexus palsy, and pressure necrosis to either the face or chest wall [11]. No neurological complications were observed in our study.

According to our results, there were no significant differences between the onset time of sensory and motor blockade, but recovery time of the sensory and motor blockade was shorter in the levobupivacaine group, and further, the maximum level of sensory block was higher in this group. In contrast, Fattorini et al. [12] and Sathitkarnmanee et al. [13] demonstrated that the intrathecal administration of $3 \mathrm{ml} 0.5 \%$ levobupivacaine or racemic bupivacaine in two different groups of patients provided no significant differences with regard to either anesthetic or hemodynamic characteristics. They found no significant differences between bupivacaine and levobupivacaine in the characteristics of subarachnoid blockade. In Fattorini et al.'s [12] study of lower limb major surgery, they applied spinal block in the sitting position, and patients were placed in the supine position, and then the patients were placed in lateral positions for limb surgery. Sathitkarnmanee et al. [13] performed different surgical procedures and changed the patients' position according the surgical area. In our study, spinal anesthesia was performed with the patient in the left lateral decubitus position, and after injection, patients turned to the supine position immediately, and then all patients were put into the prone position. In Cuvas's [6] study, which was also performed in the prone position, the characteristics of sensory and motor blocked were similar in the bupivacaine and levobupivacaine groups. But they used low- 
dose isobaric local anesthetic and spinal anesthesia was performed with the patient in sitting position and operations performed in the standard prone position. In our study, surgery performed with the patient in the kneeelbow position. The position and type of surgery may have caused the differences in the characteristics of spinal anesthesia and block level noted between studies.

Gautier et al. [14] described a combined spinal-epidural technique, isobaric bupivacaine $8 \mathrm{mg}$, levobupivacaine $8 \mathrm{mg}$ or ropivacaine $12 \mathrm{mg}$, each combined with sufentanil $2.5 \mathrm{mg}$. Anesthesia was effective in the bupivacaine group. Bupivacaine $8 \mathrm{mg}$ was associated with a significantly superior success rate to that observed in the levobupivacaine group $(p<0.05)$. It also provided a longer duration of analgesia and motor block ( $p<0.05$ vs. levobupivacaine and ropivacaine). However, in our study, we used only isobaric local anesthetics, and did not combine any opioid with local anesthetic for subarachnoid blockade.

Glaser et al. [15] compared $17.5 \mathrm{mg}$ of $0.5 \%$ levobupivacaine and racemic bupivacaine for hip replacement, observing a substantial equality in the pharmacological and cardiovascular characteristics of these isomers. In one study, higher plasma concentrations of levobupivacaine, the plasma concentration of free (unbound) levobupivacaine is lower than that of bupivacaine racemate because of greater protein binding of the levorotatory enantiomer [16]. Levobupivacaine has less toxic effects on cardiovascular and the central nervous system [17], so it is an alternative choice.

Burke et al. [18] in a non-comparative study used $15 \mathrm{mg}$ of $0.5 \%$ levobupivacaine for elective lower limb surgery in a small group of patients. The quality of anesthesia was adequate in only $90 \%$ of cases. Vercauteren et al. [19] used $2 \mathrm{ml}$ of $0.125 \%$ levobupivacaine or racemic bupivacaine for spinal-epidural analgesia in labor. They found similar clinical effects except that levobupivacaine produced no motor block, while motor block equivalent to grade I Bromage score as compared to $34 \%$ of patients in the bupivacaine group. In our study, we found no significant differences in the onset time of motor block, but mobilization time was earlier in the levobupivacaine group.

Cox et al. [20] compared epidural $0.5 \%$ levobupivacaine or bupivacaine for lower limb surgery. They found a trend towards less motor block with an equivalent dose of levobupivacaine. We found that both sensorial and motor block recovery times were shorter in the levobupivacaine group. Lee et al. [21] suggested that $0.5 \%$ levobupivacaine is an effective alternative to racemic bupivacaine in spinal anesthesia for surgery that requires a sensory block to at least T10. Regarding the rate of onset and extent of the sensory block to pinprick, Luck et al. [22] showed no significant difference among ropivacaine, levobupivacaine and bupivacaine with respect to the onset time to T10, maximum extent of cephalic spread, and the time to maximum spread. In our study, there were no statistically significant differences between the onset times of sensory blockade but the time to two-segment regression of sensory blockade was earlier in the levobupivacaine group.

Kokki et al. [23] demonstrated that the clinical characteristics of intrathecal levobupivacaine in young children are fairly similar to those obtained with racemic bupivacaine at the same dose. Levobupivacaine has very similar pharmacokinetic properties to those of racemic bupivacaine. Cardiovascular collapse can still occur with both bupivacaine and levobupivacaine with accidental intravascular injections, but the potential for toxicity is less with levobupivacaine than with racemic bupivacaine [24].

Taspinar et al. [25] compared equipotent doses of ropivacaine and levobupivacaine regarding walk-out criteria and the characteristics of spinal anesthesia in inguinal herniorrhaphy surgery. Similar to our results, they found sensory block onset time was significantly shorter in the levobupivacaine group. Time to home discharge was shorter in the levobupivacaine group, but this difference was not statistically significant in their study. All of our patients went home 1 day after the surgery.

In our study, we compared satisfaction scores of the surgeon and patients. Fattorini et al. [12], Casati et al. [26] and Vanna et al. [27] found no significant differences between satisfaction scores of surgeons, and Cuvas et al. [6] found no statistical difference between bupivacaine and levobupivacaine in terms of patient and surgeon satisfaction scores. In our study, there were no significant differences between groups regarding patients' scores. But the surgeon's satisfaction score was higher. The surgeon emphased that recovery times of sensory and motor blockade were shorter in the levobupivacaine group, and the surgeon was able to conduct a postoperative neurological exam earlier in the levobupivacaine group.

In conclusion, our results showed that recovery of sensory and motor blockade was shorter in the levobupivacaine group than in the bupivacaine group. Mobilization was earlier with levobupivacaine, which suggests that this can be an advantage for postoperative recovery; it is also an advantage for the surgeon to be able to examine the patient sooner and an advantage for the patient in terms of early discharge. We think that the clinical efficiency of levobupivacaine is better and the recovery from spinal anesthesia is quicker when compared to bupivacaine. Based on these data, levobupivacaine seems to be a safe alternative for lumbar disc surgery under spinal anesthesia.

Conflict of interest None. 


\section{References}

1. Hassi N, Badaoui R, Cagny-Bellet A, Sifeddine S, Ossart M (1995) Spinal anesthesia for disk herniation and lumbar laminectomy. Apropos of 77 cases. Cah Anesthesiol 43:21-25

2. Yilmaz C, Buyrukcu SO, Cansever T, Gulsen S, Altinors N, Caner H (2010) Lumbar microdiscectomy with spinal anesthesia: comparison of prone and knee-chest positions in means of hemodynamic and respiratory function. Spine (Phila Pa 1976) 35:1176-1184. doi:10.1097/BRS.0b013e3181be5866

3. Dripps RD, Vandam LD (1954) Long-term follow-up of patients who received 10,098 spinal anesthetics: failure to discover major neurological sequelae. J Am Med Assoc 156:1486-1491

4. Phillips OC, Ebner H, Nelson AT, Black MH (1969) Neurologic complications following spinal anesthesia with lidocaine: a prospective review of 10,440 cases. Anesthesiology 30:284-289

5. Nazon D, Abergel G, Hatem CM (2003) Critical care in orthopedic and spine surgery. Crit Care Clin 19:33-53

6. Cuvas O, Gulec H, Karaaslan M, Basar H (2009) The use of low dose plain solutions of local anaesthetic agents for spinal anaesthesia in the prone position: bupivacaine compared with levobupivacaine. Anaesthesia 64:14-18. doi:10.1111/j.13652044.2008.05680.x

7. Gouveia MA, Imbelloni LE (2006) Understanding spinal anesthesia. Acta Anaesthesiol Scand 50:259-260. doi:10.1111/j.13996576.2006.00895.x

8. Sanford M, Keating GM (2010) Levobupivacaine: a review of its use in regional anaesthesia and pain management. Drugs 70:761-791. doi:10.2165/11203250-000000000-00000

9. Bromage PR (1965) A comparison of the hydrochloride and carbon dioxide salts of lidocaine and prilocaine in epidural analgesia. Acta Anaesthesiol Scand Suppl 16:55-69

10. Tetzlaff JE, Dilger JA, Kodsy M, Al-Bataineh J, Yoon HJ, Bell GR (1998) Spinal anesthesia for elective lumbar spine surgery. J Clin Anesth 10:666-669

11. Sadrolsadat SH, Mahdavi AR, Moharari RS, Khajavi MR, Khashayar P, Najafi A, Amirjamshidi A (2009) A prospective randomized trial comparing the technique of spinal and general anesthesia for lumbar disk surgery: a study of 100 cases. Surg Neurol 71:60-65. doi:10.1016/j.surneu.2008.08.003 (discussion 65)

12. Fattorini F, Ricci Z, Rocco A, Romano R, Pascarella MA, Pinto G (2006) Levobupivacaine versus racemic bupivacaine for spinal anaesthesia in orthopaedic major surgery. Minerva Anestesiol 72:637-644

13. Sathitkarnmanee T, Thongrong $\mathrm{C}$, Tribuddharat $\mathrm{S}, \mathrm{Bn}$ MT, Bn KP, Bn RK (2011) A comparison of spinal isobaric levobupivacaine and racemic bupivacaine for lower abdominal and lower extremity surgery. J Med Assoc Thai 94:716-720
14. Gautier P, De Kock M, Huberty L, Demir T, Izydorczic M, Vanderick B (2003) Comparison of the effects of intrathecal ropivacaine, levobupivacaine, and bupivacaine for Caesarean section. Br J Anaesth 91:684-689

15. Glaser C, Marhofer P, Zimpfer G, Heinz MT, Sitzwohl C, Kapral S, Schindler I (2002) Levobupivacaine versus racemic bupivacaine for spinal anesthesia. Anesth Analg 94:194-198 (Table of contents)

16. Verghese ST, Hannallah RS (2010) Acute pain management in children. J Pain Res 3:105-123

17. Casati A, Putzu M (2005) Bupivacaine, levobupivacaine and ropivacaine: are they clinically different? Best Pract Res Clin Anaesthesiol 19:247-268

18. Burke D, Kennedy S, Bannister J (1999) Spinal anesthesia with $0.5 \% \mathrm{~S}(-)$-bupivacaine for elective lower limb surgery. Reg Anesth Pain Med 24:519-523

19. Vercauteren MP, Hans G, De Decker K, Adriaensen HA (2001) Levobupivacaine combined with sufentanil and epinephrine for intrathecal labor analgesia: a comparison with racemic bupivacaine. Anesth Analg 93:996-1000

20. Cox CR, Faccenda KA, Gilhooly C, Bannister J, Scott NB, Morrison LM (1998) Extradural S(-)-bupivacaine: comparison with racemic RS-bupivacaine. Br J Anaesth 80:289-293

21. Lee YY, Muchhal K, Chan CK (2003) Levobupivacaine versus racemic bupivacaine in spinal anaesthesia for urological surgery. Anaesth Intensive Care 31:637-641

22. Luck JF, Fettes PD, Wildsmith JA (2008) Spinal anaesthesia for elective surgery: a comparison of hyperbaric solutions of racemic bupivacaine, levobupivacaine, and ropivacaine. $\mathrm{Br} \mathrm{J}$ Anaesth 101:705-710. doi:10.1093/bja/aen250

23. Kokki H, Ylonen P, Heikkinen M, Reinikainen M (2004) Levobupivacaine for pediatric spinal anesthesia. Anesth Analg 98:64-67 (Table of contents)

24. Foster RH, Markham A (2000) Levobupivacaine: a review of its pharmacology and use as a local anaesthetic. Drugs 59:551-579

25. Taspinar V, Sahin A, Donmez NF, Pala Y, Selcuk A, Ozcan M, Dikmen B (2011) Low-dose ropivacaine or levobupivacaine walking spinal anesthesia in ambulatory inguinal herniorrhaphy. J Anesth 25:219-224. doi:10.1007/s00540-010-1089-9

26. Casati A, Moizo E, Marchetti C, Vinciguerra F (2004) A prospective, randomized, double-blind comparison of unilateral spinal anesthesia with hyperbaric bupivacaine, ropivacaine, or levobupivacaine for inguinal herniorrhaphy. Anesth Analg 99:1387-1392. doi:10.1213/01.ANE.0000132972.61498.F1 table of contents

27. Vanna O, Chumsang L, Thongmee S (2006) Levobupivacaine and bupivacaine in spinal anesthesia for transurethral endoscopic surgery. J Med Assoc Thai 89:1133-1139 